\title{
CÁLCULO DO PONTO DE PEDIDO BASEADO EM PREVISÕES DE UMA POLÍTICA $<Q, r>$ DE GESTÃO DE ESTOQUES
}

\author{
Eduardo Saggioro Garcia \\ Visagio Consultoria Assessoria e Desenvolvimento Ltda \\ Rio de Janeiro - RJ \\ eduardo.saggioro@visagio.com.br \\ Virgílio José Martins Ferreira Filho* \\ Engenharia de Produção \\ Universidade Federal do Rio de Janeiro (UFRJ) \\ Rio de Janeiro - RJ \\ virgilio@ufrj.br \\ * Corresponding author / autor para quem as correspondências devem ser encaminhadas \\ Recebido em 07/2005; aceito em 04/2007 após 1 revisão \\ Received July 2005; accepted April 2007 after one revision
}

\begin{abstract}
Resumo
Um das políticas de gestão de estoques mais conhecidas e utilizadas é o sistema de lote econômico com ponto de pedido, também conhecido como sistema $\langle Q, r\rangle$. O objetivo deste artigo é apresentar uma nova abordagem para o cálculo do ponto de pedido, baseada na previsão da demanda para cada período durante o tempo de ressuprimento. Erros de previsão e tempos de ressuprimento são as variáveis aleatórias consideradas sendo o ponto de pedido calculado com base na distribuição composta desses parâmetros. Simulações são realizadas para avaliar a adequação do modelo proposto, sob condições tais como: diferentes distribuições do lead-time, da demanda e diferentes magnitudes do erro de previsão. A experimentação conduzida procura avaliar tanto a validade do modelo teórico proposto quanto os possíveis desvios das premissas consideradas, principalmente a da demanda ser considerada normalmente distribuída no lead-time.
\end{abstract}

Palavras-chave: estoques; ponto de pedido; previsão; simulação.

\begin{abstract}
One of the most known policy of inventory management is the order quantity and reorder point, or $<Q, r>$ policy. The purpose of this paper is to present a new analytical approach for evaluating reorder points in inventory management policies. This approach considers the reorder point as a function of forecasted demands in the planning horizon, which can vary through time. Lead-times and forecast errors are the uncertainties present in the model. The composed distribution of these parameters is used to obtain an analytical expression for the proposed forecast based reorder point. Simulation experiments are performed in order to validate and test the accuracy of the proposed model. The experiments consider different lead-time and demand distributions and also different magnitude of forecast errors. Beyond the accuracy of the analytical model the simulations seeks to evaluate the premises adopted, mainly the one of normally distributed lead-time demand.
\end{abstract}

Keywords: inventory; reorder point; forecast; simulation. 


\section{Introdução}

O gerenciamento dos estoques, uma das questões mais antigas da administração, ainda desperta muito interesse, seja no dia a dia das organizações seja no meio acadêmico. Depois de um período em que foram desenvolvidos vários modelos analíticos para abordar a questão dos estoques seguiu-se uma época em que estes modelos foram relegados a segundo plano prevalecendo abordagens de gestão da produção que preconizavam sistemas sincronizados com minimização/eliminação dos estoques. Mais recentemente, sistemas mais complexos envolvendo cadeias de suprimento mais longas e portanto sujeitas a maior variabilidade vem requerendo modelos analíticos que permitam tratar a questão dos estoques ao longo da cadeia.

As questões principais no gerenciamento de estoques, sejam em grandes cadeias, sejam em uma organização isolada, continuam sendo quando e quanto repor levando em conta as possíveis variabilidades tanto no suprimento quanto na demanda. Um das políticas de gestão de estoques mais conhecidas e utilizadas é o sistema de lote econômico com ponto de pedido, também conhecido como sistema $\langle Q, r\rangle$. Neste modelo, uma quantidade $Q$ é ordenada assim que o estoque disponível atinge o patamar de $r$ unidades, chamado de ponto de pedido ou nível de reposição. A quantidade $Q$ é usualmente determinada pela expressão do Lote Econômico de Ressuprimento ou por algum outro modelo de otimização, como pode ser visto em livros clássicos de gestão de estoques como Hadley \& Within (1963) e Brown (1967), ou em livros mais recentes como Silver, Rycke \& Peterson (1998).

Já o ponto de pedido $r$ se baseia em atingir algum padrão de nível de serviço, tendo em consideração a variabilidade estocástica da demanda total durante o tempo de ressuprimento, também conhecida como demanda no lead-time. Embora existam muitas maneiras de se definir nível de serviço, como disponibilidade de produto (fill rate), uma abordagem comum é considerá-lo como a probabilidade de não ocorrência de uma ruptura de estoques (stockout) durante o tempo de ressuprimento.

Com base no nível de serviço desejado, $r$ é em geral calculado como a demanda média no lead-time mais um estoque de segurança. Para o cálculo apropriado deste estoque de segurança deve-se conhecer a distribuição de probabilidades da demanda no lead-time, que é uma distribuição composta definida pela convolução de um número aleatório de períodos, cada qual apresentando uma demanda aleatória. Alternativamente, outro método usual de calcular o estoque de segurança é utilizando a previsão da demanda no lead-time, juntamente com os respectivos erros de previsão.

O objetivo deste artigo é apresentar uma nova abordagem para o cálculo do ponto de pedido, baseada na previsão da demanda para cada período durante o tempo de ressuprimento. Erros de previsão e tempos de ressuprimento são as variáveis aleatórias consideradas sendo o ponto de pedido calculado com base na distribuição composta desses parâmetros. Simulações são realizadas para avaliar a adequação do modelo proposto, sob condições tais como: diferentes distribuições do lead-time, da demanda e diferentes níveis de erro de previsão. A experimentação conduzida procura avaliar tanto a validade do modelo teórico proposto, quanto os possíveis desvios das premissas consideradas, principalmente a da demanda ser considerada normalmente distribuída no lead-time.

Na sequência do artigo é apresentada uma breve revisão bibliográfica discutindo as premissas da formulação clássica, as quais são apresentadas e analisadas como fundamentos para o desenvolvimento do modelo proposto na seção 3 . Na seção 4 é apresentada a 
metodologia de simulação desenvolvida para validar o modelo, bem como o processo de experimentação conduzido. A seção 5 conclui o trabalho, apresentando também possíveis formas de utilização da modelagem proposta.

\section{Análise e premissas da formulação clássica do modelo de ponto de pedido}

Nos modelos de estoques existentes na literatura e usados nas organizações, diversas são as premissas assumidas, muitas vezes de modo implícito. Estas premissas têm efeitos tanto nos custos quanto no nível de serviço. Algumas destas suposições, apontadas por exemplo em Silver (1981) ou em Mentzer \& Krishnan (1988) são: (i) a distribuição da demanda no tempo de serviço tem uma forma determinada (na maioria das vezes é assumida a distribuição normal) (ii) os parâmetros, principalmente média e desvio-padrão, da distribuição são conhecidos, (iii) a distribuição da demanda no lead-time é uma composição de uma distribuição contínua para a demanda com uma distribuição discreta do lead-time e além disto que a demanda é independente do lead-time, (iv) a cada demanda diária apresenta a mesma média e o mesmo desvio-padrão e ainda é admitido que não existe autocorrelação entre as demandas diárias. Diversos artigos presentes na literatura discutem os efeitos destas premissas.

A simplificação mais comum é considerar que a demanda no lead-time segue uma distribuição normal (veja por exemplo Eppen \& Martin, 1988). Com esta suposição, para um dado nível de serviço, basta estimar a média e o desvio-padrão da demanda no lead-time para se determinar $r$. Porém, segundo Tyworth (1992), mesmo que o tempo de ressuprimento e as demandas em cada período sejam normalmente distribuídos, a distribuição composta não segue uma distribuição normal. Apesar disso, o pressuposto de normalidade é vastamente usado como simplificação do problema, como visto em vários livros de logística e gestão de materiais como Bowersox \& Closs (1996) e Ballou (2001). Lau (1989), além de analisar o efeito da consideração da distribuição normal, apresenta um método para calcular níveis de serviço e os correspondentes pontos de pedido com base nos quatro primeiros momentos (média, variância, assimetria e curtose) de uma dada distribuição de demanda no lead-time.

A segunda categoria de premissas é via de regra suportada pela existência de dados históricos. Na ausência destes, estimativas podem ser feitas principalmente para as médias da demanda e do lead-time, entretanto, como argumentam Lariviere \& Porteus (1999), outros momentos como variância, assimetria e curtose não são fáceis de avaliar intuitivamente. Destes momentos, a variância, mesmo nos modelos mais simplificados, é de extrema relevância no cálculo do ponto de pedido. Zipkin (2001) e Wanke \& Saliby (2005), são alguns dos autores que se preocupam com a terceira categoria de premissas. Os últimos desenvolvem um modelo que considera uma distribuição uniforme para a demanda e para o lead-time.

A quarta categoria de premissas consiste em considerar que, durante o tempo de ressuprimento, as demandas em cada período seguem distribuições de probabilidade idênticas, isto é, são variáveis aleatórias com a mesma média e o mesmo desvio-padrão. Desta forma, se uma parte da variabilidade da demanda pode ser prevista, a expressão clássica do estoque de segurança tende a dar valores superestimados, resultando em maiores custos de carregar estoques. Krupp (1997) tenta resolver parte deste problema formulando um modelo para o cálculo do estoque de segurança baseado nos erros percentuais de previsão. Desta maneira, se é possível prever variabilidades na demanda ao longo do tempo,

Pesquisa Operacional, v.29, n.3, p.605-622, Setembro a Dezembro de 2009 
como padrões cíclicos, tendências e sazonalidades, os estoques de segurança são determinados de forma mais eficiente. Este modelo entretanto não contempla aleatoriedade nos tempos de ressuprimento, sendo portanto pouco aplicável a muitas situações reais. Outra premissa dentro desta categoria é a suposição de que não existe autocorrelação entre as demandas diárias. Zinn, Marmorstein \& Charnes (1992) analisam o efeito de demandas autocorrelacionadas, mostrando como este fato pode afetar os níveis de serviço projetados.

A seguir é apresentada a formulação clássica da política $\langle Q, r\rangle$, com base na seguinte notação.

Sejam:

$Q-\quad$ tamanho do pedido,

$r-\quad$ ponto de pedido,

$d_{t}-\quad$ variável aleatória - demanda no período $t$,

$L$ - variável aleatória - tempo de ressuprimento (lead-time),

$L_{\text {min }}$ - valor mínimo que o lead-time pode assumir,

$L_{\max }$ - valor máximo que o lead-time pode assumir,

$D L$ - variável aleatória - demanda no lead-time,

$S S$ - estoque de segurança,

$k$ - fator de segurança para se atingir o nível de serviço desejado, com base na distribuição normal unitária,

$\mu_{X}$ - média da variável aleatória $X$ (por exemplo, $X=D L$ ),

$\sigma_{X}$ - desvio-padrão da variável aleatória $X$,

$p_{t}(L)$ - probabilidade que o lead-time $t$ assuma um valor igual a $L$.

O ponto de pedido, considerando as premissas apresentadas no início da seção, é dado por:

$$
\begin{aligned}
& r=\mu_{D L}+S S \\
& S S=k \sigma_{D L}
\end{aligned}
$$

No que se refere a $r$, deve-se primeiramente perceber que este depende dos possíveis valores que pode assumir a demanda no lead-time, $D L$. Esta é a soma de cada demanda por unidade de tempo, $d_{t}$, durante o lead-time de ressuprimento $L$, isto é:

$$
D L=\sum_{t=1}^{L} d_{t}
$$

Para se poder calcular o ponto de pedido é necessário obter a média e o desvio-padrão da demanda no lead-time. Uma possibilidade é coletar dados históricos e calcular diretamente as estatísticas necessárias. Então as estatísticas de $D L$ são dadas por:

$$
\begin{aligned}
& \mu_{D L}=\sum_{L=L \min }^{L \max }\left(p_{t}(L) \sum_{t=1}^{L} \mu_{d_{t}}\right) \\
& \sigma_{D L}^{2}=\sum_{L=L \min }^{L \max } p_{t}(L)\left[\left(\sum_{t=1}^{L}{\sigma_{d_{t}}}^{2}\right)+\left(\mu_{D L}-\sum_{t=1}^{L} \mu_{d_{t}}\right)^{2}\right]
\end{aligned}
$$


Entretanto, o modo mais usual de se obter a média e os desvio-padrão da demanda no leadtime $D L$ é usando as estatísticas da demanda por unidade de tempo e do lead-time. Sob a hipótese de que a demanda no lead-time siga uma distribuição normal e ainda que cada demanda $d_{t}$ tenha a mesma média e desvio-padrão,

$$
\begin{aligned}
& \mu_{D L}=\mu_{d} \mu_{L} \\
& \sigma_{D L}^{2}=\mu_{L} \sigma_{d}^{2}+\mu_{d}^{2} \sigma_{L}^{2}
\end{aligned}
$$

Uma vez definido o modelo clássico pode-se analisar o significado dos seus termos. A variância da demanda no lead-time $\sigma_{D L}^{2}$, é a soma de dois termos: o primeiro é devido a aleatoriedade da demanda diária $d_{i}$ enquanto o segundo é devido a aleatoriedade do leadtime. O resultado expresso em (7) é derivado diretamente da teoria das probabilidades (veja, por exemplo Meyer (1965).

A expressão (7) pode ser simplificada se é assumido que o lead-time $L$ é determinístico.

$$
\sigma_{D L}^{2}=\mathrm{L} \sigma_{d}^{2}
$$

Ela pode também ser generalizada para considerar distribuições contínuas do lead-time. Veja, por exemplo, Wanke \& Saliby (1995), Lau (1989).

$$
\sigma_{D L}^{2}=\mu_{L} \sigma_{d}^{2}+\mu_{d}^{2} \sigma_{L}^{2}+\frac{\sigma_{d}^{2} \sigma_{L}^{2}}{\mu_{L}}
$$

No ultimo termo da expressão (9), o produto de variâncias dividido pela média do lead-time é geralmente pequeno quando comparado com os demais termos, assim este termo é muitas vezes negligenciado na maioria dos livros de gestão de materiais, como por exemplo Bowersox et al. (1996), Ballou (2001), Garcia, Reis et al. (2006).

\section{Desenvolvimento de um ponto de pedido baseado em previsões}

Em Zinn \& Marmorstein (1990) é demonstrado que, até mesmo para o modelo clássico, se erros de previsão são considerados ao invés da variabilidade da demanda os estoques de segurança podem ser reduzidos sem reduzir os níveis de serviço ao cliente. Estes autores enfatizam que na expressão clássica do ponto de pedido está implícito que as previsões para cada período são a demanda média, o que pode não ser uma suposição válida em muitas situações reais.

Agora, baseado nas equações apresentadas na seção anterior será desenvolvido um modelo para o cálculo do ponto de pedido baseado em previsões. Primeiramente deve-se estabelecer uma forma de mensurar as diferenças entre as demandas e suas respectivas previsões. A variável $e_{t}$ é então definida como a razão entre a demanda para o período $t$ e sua previsão $f_{t}$.

$$
\begin{aligned}
& e_{t}=\frac{d_{t}}{f_{t}} \\
& d_{t}=e_{t} f_{t}
\end{aligned}
$$


A variável $e_{t}$ é uma forma de se mediar a acurácia da previsão. Tendo em vista que as previsões $f_{t}$ são parâmetros determinísticos e as demandas $d_{t}$ variáveis aleatórias, cada $e_{t}$ é também uma variável aleatória. A demanda no Lead- time pode ser agora escrita como:

$$
D L=\sum_{t=1}^{L} f_{t} \cdot e_{t}
$$

Como apresentado na expressão (1), para determinar o ponto de pedido $r$ é necessário calcular a média e o desvio-padrão de $D L$. Seja $\mu_{e_{t}}$ e $\sigma_{e_{t}}$ respectivamente a média e o desvio-padrão de cada variável aleatória $e_{t}$. É interessante notar que caso não haja viés nas previsões, $\mu_{e_{t}}$ será igual a 1 . Se as previsões estiverem sistematicamente acima das demandas $\mu_{e_{t}}$ assumirá valores menores que 1 , e se as previsões estiverem sistematicamente abaixo das demandas $\mu_{e_{t}}$ assumirá valores maiores que 1. Combinando as expressões (4), (5) e (12) a média e o desvio-padrão da demanda no lead-time podem ser calculados respectivamente por:

$$
\begin{aligned}
& \mu_{D L}=\sum_{L=L \min }^{L \max }\left(p_{t}(L) \sum_{t=1}^{L} f_{t} \mu_{e_{t}}\right) \\
& \sigma_{D L}^{2}=\sum_{L=L \min }^{L \max } p_{t}(L)\left[\left(\sum_{t=1}^{L} f_{t}^{2} \sigma_{e_{t}}{ }^{2}\right)+\left(\mu_{D L}-\sum_{t=1}^{L} f_{t} \mu_{e_{t}}\right)^{2}\right]
\end{aligned}
$$

Se o lead-time $L$ é determinístico, as expressões (14)-(15) se reduzem a:

$$
\begin{gathered}
\mu_{D L}=\sum_{t=1}^{L} f_{t} \mu_{e_{t}} \\
\sigma_{D L}^{2}=\sum_{t=1}^{L} f_{t}^{2} \sigma_{e_{t}}^{2}
\end{gathered}
$$

O ponto de pedido baseado em previsões, chamado aqui de $r_{f}$, como o seu equivalente clássico $r$, pode ser escrito como função da média e do desvio-padrão da demanda no leadtime $D L$.

$$
r_{f}=\mu_{D L}+k \sigma_{D L}
$$

Mais uma vez, a constante $k$, baseada na distribuição normal unitária, é o fator de segurança para se atingir um certo nível de serviço. Embora o pressuposto de normalidade da demanda no lead-time tenha sido mantido, o modelo baseado em previsões apresenta uma série de vantagens quando comparado ao modelo clássico. Como exemplos de vantagens estão: consideração de variações não aleatórias da demanda, possibilidade da magnitude dos erros de previsão variar no tempo e consideração do efeito de viés nas previsões. 


\section{Uso de simulação para validação do modelo}

Garcia, Silva \& Saliby (2002) mostram que modelos de simulação estocástica são muito úteis para validar e testar a adequação de expressões analíticas para o cálculo de estoques de segurança. No presente artigo, simulações são feitas para testar as equações propostas para $\mu_{D L}$ e $\sigma_{D L}$, e para avaliar a aderência da premissa de $D L$ normalmente distribuída. Desta forma, experimentos foram realizados. Em todos eles foram feitas 5 rodadas de simulação, cada qual com 5000 iterações, utilizando amostragem por Hipercubo Latino (Banks, 1999). Valores simulados da demanda no lead-time $D L$ foram coletadas para o cálculo de suas estatísticas. Os passos da simulação realizada em MS-Excel foram, de forma esquemática, os seguintes:

1. Geração aleatória de 5x5.000 lead-time com distribuição discreta (dada pela Tabela 1).

2. Para cada dia do lead-time gerar uma demanda diária normalmente distribuída, com média igual à previsão e desvio igual ao desvio de previsão.

3. Determinação da demanda no lead-time, através da soma dos resultados obtidos na etapa (2).

4. Cálculo das estatísticas de $D L$ (média e desvio-padrão).

5. Determinação da condição de stockout em cada um dos suprimentos, ou seja, se demanda no lead-time de suprimento $<=r$, não houve falta, caso contrário, houve falta.

6. Comparação dos resultados obtidos nas etapas (4) e (5) com as fórmulas (13) e (14) apresentadas na seção 3 .

\section{O projeto de experimentos}

De forma a avaliar a metodologia proposta sob diferentes condições foram considerados três diferentes distribuições para o lead-time (apresentadas na Tabela 1), três diferentes magnitudes de erro de previsão ( $\sigma_{e_{t}}=0,1 ; 0,3$ e 0,5 respectivamente) e três conjuntos de previsão, com características distintas (apresentadas na Tabela 2). Em todos os experimentos foi considerado previsão sem viés, ou seja, $\mu_{e_{t}}$ é igual a 1 para todos os $t$ períodos. Outro pressuposto considerado é que os $e_{t}$ são normalmente distribuídos. Além disso, foi considerado que o erro percentual de previsão é constante ao longo do tempo (valores absolutos dos erros entretanto variam no tempo, de acordo com a variabilidade das previsões). Em algumas situações é interessante considerar que os erros percentuais de previsão crescem à medida que o horizonte temporal se distancia. Entretanto, é assumido nos experimentos que o horizonte de planejamento não é amplo o suficiente para gerar diferenças consideráveis na previsibilidade das demandas.

Como pode ser visto na Tabela 1 e na Figura 1 três distribuições para o lead-time foram testadas uma primeira que se assemelha à distribuição normal, uma distribuição uniforme e uma distribuição com probabilidades máximas nos extremos. Em todos os casos o lead-time mínimo $L_{\min }$ é de 3 períodos, o máximo $L_{\max }$ é de 9 períodos, o valor médio de $L$ é de 6 períodos os desvios-padrão são de respectivamente 1,428; 2 e 2,588.

Pesquisa Operacional, v.29, n.3, p.605-622, Setembro a Dezembro de 2009 
Tabela 1 - Distribuições de Probabilidade para o Lead-time L.

\begin{tabular}{|c|c|c|c|}
\hline & $\begin{array}{c}\text { Lead-time } \\
\text { S-Normal }\end{array}$ & $\begin{array}{c}\text { Lead-time } \\
\text { Uniforme }\end{array}$ & $\begin{array}{c}\text { Lead-time } \\
\text { 2-Extremos }\end{array}$ \\
\hline$L$ & $p_{t}(L)$ & $p_{t}(L)$ & $p_{t}(L)$ \\
\hline 3 & $4 \%$ & $14 \%$ & $30 \%$ \\
\hline 4 & $11 \%$ & $14 \%$ & $15 \%$ \\
\hline 5 & $22 \%$ & $14 \%$ & $5 \%$ \\
\hline 6 & $26 \%$ & $14 \%$ & $0 \%$ \\
\hline 7 & $22 \%$ & $14 \%$ & $5 \%$ \\
\hline 8 & $11 \%$ & $14 \%$ & $30 \%$ \\
\hline 9 & $4 \%$ & $14 \%$ & 6 \\
\hline$\mu_{L}$ & 6 & 6 & 2,588 \\
\hline$\sigma_{L}$ & 1,428 & 2 & \\
\hline
\end{tabular}
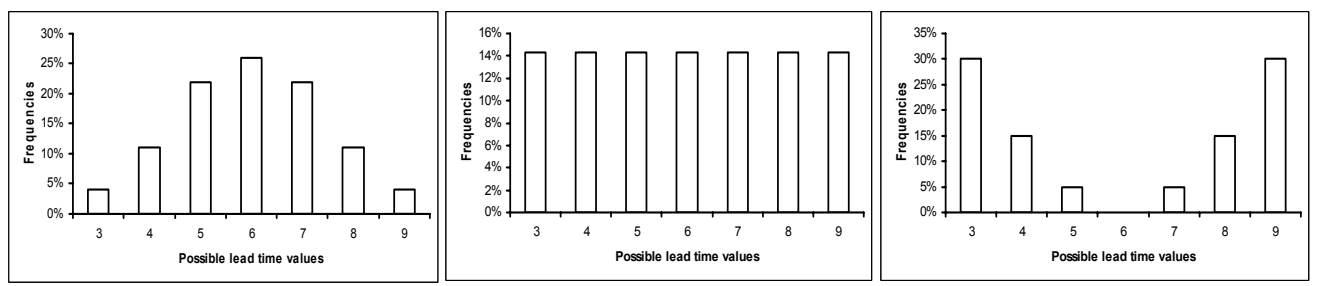

Figura 1 - Histograma das Distribuições de Probabilidade para o Lead-time L.

No que diz respeito à previsão de demanda, foram simuladas três situações apresentadas na Tabela 2. A primeira corresponde a uma situação onde a previsão é constante para todos os períodos de tempo. Para as demais situações considerou-se a ocorrência de sazonalidade baixa e alta. O propósito destes experimentos é testar se grandes variações na previsão têm impactos significativos no nível de serviço aos clientes.

Tabela 2 - Previsões para a Demanda ao Longo do Horizonte de Planejamento.

\begin{tabular}{|c|c|c|c|}
\hline & $\begin{array}{c}\text { Previsão sem } \\
\text { sazonalidade }\end{array}$ & $\begin{array}{c}\text { Previsão com baixa } \\
\text { sazonalidade }\end{array}$ & $\begin{array}{c}\text { Previsão com alta } \\
\text { sazonalidade }\end{array}$ \\
\hline Período $t$ & $f_{t}$ & $f_{t}$ & $f_{t}$ \\
\hline 1 & 100 & 100 & 100 \\
\hline 2 & 100 & 125 & 130 \\
\hline 3 & 100 & 75 & 75 \\
\hline 4 & 100 & 130 & 160 \\
\hline 5 & 100 & 105 & 40 \\
\hline 6 & 100 & 70 & 120 \\
\hline 7 & 100 & 115 & 55 \\
\hline 8 & 100 & 80 & 85 \\
\hline 9 & 100 & 100 & 55 \\
\hline
\end{tabular}




\section{Resultados experimentais e discussão}

Com o objetivo de ilustrar a aplicação do modelo proposto, bem como de compará-lo com o modelo clássico de ponto de pedido, primeiro serão apresentados os resultados para a situação onde todas as previsões têm o mesmo valor. Como as previsões não variam no tempo, os pressupostos considerados neste primeiro experimento são exatamente os mesmos pressupostos da formulação do modelo clássico de ponto de pedido. Dessa maneira, a média da demanda no lead-time $\mu_{D L}$ apresentada na equação (13) e a variância da demanda no lead-time $\sigma_{D L}^{2}$ apresentada na equação (14) devem dar resultados iguais aos seus equivalentes no modelo clássico.

\section{Um exemplo ilustrativo}

Sejam os dados, retirados das Tabelas 1 e 2 resumidos na Tabela 3 a seguir:

Tabela 3 - Resumo dos dados para o exemplo ilustrativo.

\begin{tabular}{|c|c|c|c|c|c|c|c|}
\hline$L$ & 3 & 4 & 5 & 6 & 7 & 8 & 9 \\
\hline$p_{t}(L)$ & $4 \%$ & $11 \%$ & $22 \%$ & $26 \%$ & $22 \%$ & $11 \%$ & $4 \%$ \\
\hline$f_{t(L)}$ & 100 & 100 & 100 & 100 & 100 & 100 & 100 \\
\hline
\end{tabular}

Assumindo ainda um erro de percentual de previsão constante ao longo do tempo com $\sigma_{e_{t}}^{2}=0,3$. Então teremos uma demanda diária com média 100 e desvio-padrão de 30 unidades de produto, isto é:

$$
\mu_{d}=100, \quad \sigma_{d}=30,
$$

além disto calculando as estatísticas diretamente da tabela

$$
\mu_{L}=6 \quad \sigma_{L}=1.428
$$

Usando as equações (6) e (7) obtemos:

$$
\mu_{D L}=6 \times 100=600, \quad \sigma_{D L}^{2}=6 \times 30^{2}+100^{2} \times 2,04=25800 \Rightarrow \sigma_{D L}=160,6238
$$

ou usando as equações (13) e (14)

$$
\mu_{D L}=\sum_{t=3}^{9} 100 \times 1=600, \quad \sigma_{D L}^{2}=\sum_{L=3}^{9} p_{t}(L)\left[L \times 100^{2} \times 0,3^{2}+(600-L \times 100)^{2}\right]=25800 .
$$

Para validar as equações propostas, a média e o desvio-padrão de $D L$ foram calculados para cada rodada de simulação. Alguns percentis de $D L$ também foram obtidos, mais especificamente os percentis $50 \%, 84,13 \%, 97,72 \%$ e 99,87\%. Estes percentis são relevantes para que seja feita a comparação entre os resultados obtidos por simulação e os valores teóricos baseados na distribuição normal. Se $D L$ é normalmente distribuído, seu percentil $50 \%$ equivale a $k$ igual a zero, seu percentil $84,13 \%$ equivale a $k$ igual a 1 , seu 
percentil 97,72\% equivale a $k$ igual a 2 e seu percentil 99,87\% equivale a $k$ igual a 3 , sendo os percentis teóricos calculados como $\mu_{D L}$ mais $k$ vezes $\sigma_{D L}$. Os resultados da simulação são mostrados na Tabela 4.

Tabela 4 - Resultados da simulação ( $1^{\circ}$ experimento).

\begin{tabular}{|c|c|c|c|c|c|c|}
\hline Corrida de simulação & $\mu_{D L}$ & $\sigma_{D L}$ & $\mathrm{P} 50_{D L}$ & $\mathrm{P} 84,13_{D L}$ & $\mathrm{P} 97,72_{D L}$ & $\mathrm{P}^{2} 99,87_{D L}$ \\
\hline 1 & 600,5 & 161,7 & 594,9 & 763,3 & 934,8 & 1065,5 \\
\hline 2 & 600,1 & 159,2 & 597,9 & 760,0 & 930,5 & 1053,1 \\
\hline 3 & 599,8 & 160,5 & 595,3 & 767,4 & 921,6 & 1073,7 \\
\hline 4 & 600,2 & 161,1 & 597,1 & 764,1 & 928,8 & 1043,7 \\
\hline 5 & 600,0 & 159,3 & 598,0 & 765,0 & 919,2 & 1051,7 \\
\hline Média das Corridas & 600,1 & 160,4 & 596,6 & 764,0 & 927,0 & 1057,5 \\
\hline Desvio das Corridas & 0,3 & 1,1 & 1,5 & 2,7 & 6,5 & 12,0 \\
\hline
\end{tabular}

Os resultados da simulação devem ser comparados aos valores teóricos (considerando as equações (13) e (14) e $D L$ normalmente distribuído). A tabela 5 apresenta estas comparações. Os valores de diferenças percentuais são calculados pela expressão

Dif. $\%=($ valor simulado - valor teórico $) /$ valor teórico.

Tabela 5 - Comparação entre resultados da simulação e valores teóricos ( $1^{\circ}$ experimento).

\begin{tabular}{|c|c|c|c|c|c|c|}
\cline { 2 - 6 } \multicolumn{1}{c|}{} & $\mu_{D L}$ & $\sigma_{D L}$ & $\mathrm{P} 50_{D L}$ & $\mathrm{P} 84,13_{D L}$ & $\mathrm{P} 97,72_{D L}$ & $\mathrm{P} 99,87_{D L}$ \\
\hline Valor teórico & 600 & 160,6 & 600 & 760,6 & 921,2 & 1081,9 \\
\hline Média das corridas & 600,1 & 160,4 & 596,6 & 764,0 & 927,0 & 1057,5 \\
\hline Diferença percentual & $-0,02 \%$ & $0,15 \%$ & $0,56 \%$ & $-0,44 \%$ & $-0,62 \%$ & $2,25 \%$ \\
\hline
\end{tabular}

Pode-se ver pela tabela 5 que os valores teóricos são bem aderentes aos resultados da simulação. Além de validar as equações (13) e (14), as comparações mostram que a consideração de $D L$ normalmente distribuído é um pressuposto válido para o caso analisado.

De forma equivalente à comparação dos percentis, uma outra maneira de visualizar a aderência do pressuposto de normalidade é analisar o percentual de vezes em que não ocorre um stockout, isto é, o número de vezes em que o valor simulado de $D L$ foi menor que o ponto de pedido baseado em previsões. Calculando $r_{f}$ pela equação (17), os percentuais de não stockouts obtidos por simulação e a comparação destes com os valores teóricos esperados para diferentes valores de k são apresentados na tabela 6 . 
Tabela 6 - Comparação entre percentuais de não stockouts simulados e teóricos ( $1^{\circ}$ experimento).

\begin{tabular}{|c|c|c|c|c|}
\hline Corrida de simulação & $\mathrm{k}=0$ & $\mathrm{k}=1$ & $\mathrm{k}=2$ & $\mathrm{k}=3$ \\
\hline 1 & $51,1 \%$ & $83,70 \%$ & $97,24 \%$ & $99,88 \%$ \\
\hline 2 & $50,62 \%$ & $84,26 \%$ & $97,34 \%$ & $99,92 \%$ \\
\hline 3 & $51,16 \%$ & $83,12 \%$ & $97,72 \%$ & $99,88 \%$ \\
\hline 4 & $50,72 \%$ & $83,60 \%$ & $97,44 \%$ & $99,96 \%$ \\
\hline 5 & $50,44 \%$ & $83,40 \%$ & $97,76 \%$ & $99,94 \%$ \\
\hline Média das corridas & $50,81 \%$ & $83,62 \%$ & $97,50 \%$ & $99,92 \%$ \\
\hline Desvio das corridas & $0,32 \%$ & $0,42 \%$ & $0,23 \%$ & $0,04 \%$ \\
\hline Valor teórico & $50,00 \%$ & $84,13 \%$ & $97,72 \%$ & $99,87 \%$ \\
\hline Diferença absoluta & $-0,81 \%$ & $0,52 \%$ & $0,22 \%$ & $-0,05 \%$ \\
\hline
\end{tabular}

A tabela 6 corrobora a conclusão baseada na tabela 5 para o caso do primeiro experimento, ou seja, o modelo analítico proposto é aderente aos pressupostos considerados em sua formulação, já que não há diferenças significativas entre valores teóricos e obtidos por simulação.

A extensão para os outros casos simulados pode ser conduzida a partir das tabelas 7 a 10 , bem como da análise dos gráficos apresentados na figura 2. Para facilitar a interpretação dos resultados eles são a seguir agrupados por objetivos pretendidos.

\section{Validação das estatísticas de $\mathrm{DL}$}

A análise das Tabelas 7 e 9 mostra que os resultados teóricos derivados das soluções analíticas obtidas na seção 3 são perfeitamente aderentes aos resultados empíricos obtidos por simulação.

(a) Análise da diferença percentual entre resultados teóricos e simulados:

(i) Com relação à média e ao desvio-padrão da demanda no lead-time as diferenças máximas entre os resultados obtidos foram de $0,15 \%$ e $0,42 \%$, respectivamente.

(ii) Com relação aos percentis P50, P84,13, P97,72 e P99,81 se observa que as diferenças entre os resultados crescem à medida que se passa do lead-time S-normal para o lead-time Uniforme e ainda mais quando se passa para o lead-time 2-Extremos.

(iii) A presença da sazonalidade na previsão não tem impacto significativo, enquanto o erro percentual de previsão tem impacto favorável, isto é, um maior erro implica em menores Dif\%.

(iv) As maiores diferenças ocorrem na cauda da distribuição, isto é para o percentil P99,72. 
(b) Análise da variância entre as corridas de simulação:

(i) O desvio-padrão das corridas é muito pequeno para a média e o desvio-padrão da demanda no lead-time (máximo de 1,3 e 2,2 respectivamente).

(ii) Da mesma maneira que ocorre com Dif\%, se observa que para os percentis P50, $\mathrm{P} 84,13, \mathrm{P} 97,72$ e $\mathrm{P} 99,81$ os desvios crescem à medida que se passa do lead-time S-normal para o lead-time uniforme e ainda mais quando se passa para o lead-time 2-Extremos.

(iii) Os desvios são menores nas situações com baixa sazonalidade e crescem com o erro de previsão.

\section{Análise da suposição de DL ser normalmente distribuída.}

Uma forma de avaliar a adequação da suposição de normalidade é computando o número de vezes que não ocorrem faltas na simulação, isto é, a percentagem de vezes em que o valor de $D L$ simulado é menor do que o ponto de pedido $r_{f}$, calculados pela expressão (17). Os resultados dos valores obtidos da simulação, para os diferentes cenários, são apresentados na Tabela 10 e nos Gráficos 2a e 2b.

Esta análise é feita observando as diferenças entre os valores obtidos por simulação para os diferentes cenários simulados e os valores teóricos obtidos para a ocorrência de falhas de suprimento (faltas) durante o lead-time.

(i) para $\mathrm{k}=0(50 \%)$ as diferenças são muito pequenas, indiferentemente à distribuição do lead-time, da ocorrência de sazonalidade e dos erros de previsão.

(ii) para $\mathrm{k}=1(84,13 \%)$ existe uma subestimação do número de faltas que é tanto maior quanto mais a distribuição do lead-time se afasta da distribuição normal. A ocorrência de sazonalidade e de erros de previsão parece não afetar a ocorrência de faltas.

(iii) para k=2 e k=3 (97,72 e 99,81\%) existe uma superestimação do número de faltas que é tanto maior quanto mais a distribuição do lead-time se afasta da distribuição normal. Novamente a ocorrência de sazonalidade e de erros de previsão parece não afetar a ocorrência de faltas.

O desvio-padrão entre as corridas de simulação é muito pequeno, inferior a 0,6.

Do conjunto de análises pode-se concluir que suposição de distribuição normal é perfeitamente adequada, sendo esta suposição mais realista para níveis baixos de serviço, para níveis de serviço muito exigentes ainda que para propósitos práticas ela possa ser assumida como válida ocorre uma ligeira superestimação das faltas. 


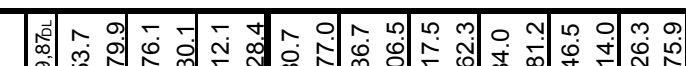

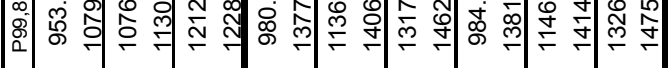

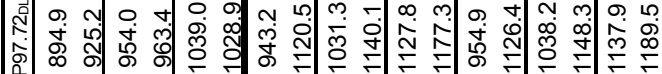
0

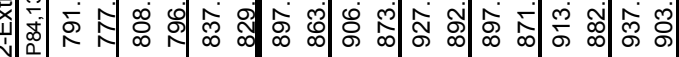
: कृष bأ

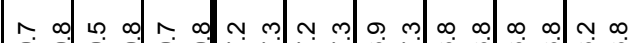

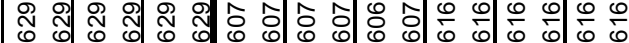

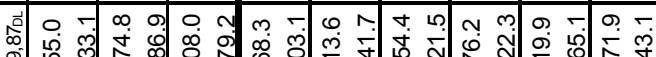

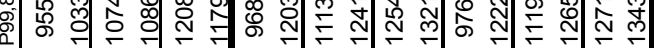

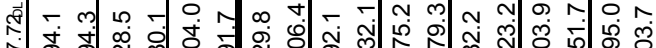

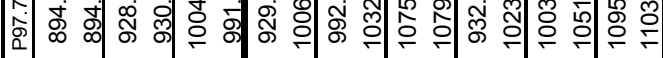

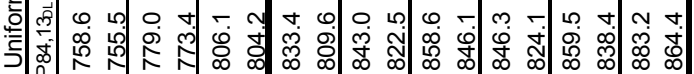
ह

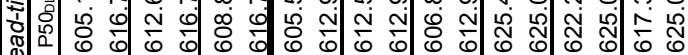

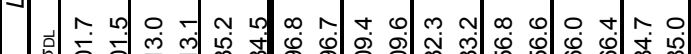

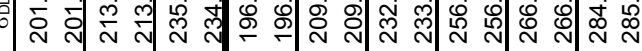

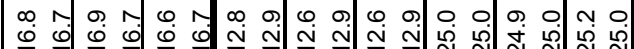

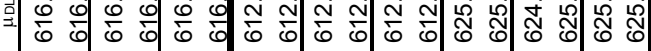

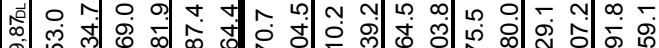
:

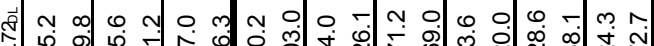
잉 ळ

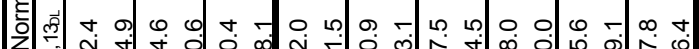

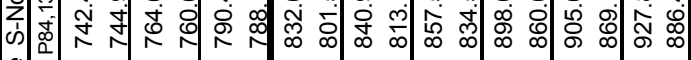
月

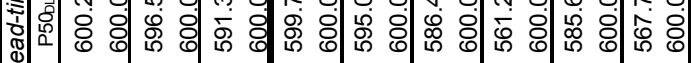
9

$\neg$ 이

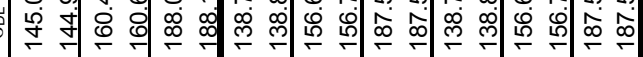

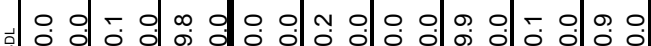

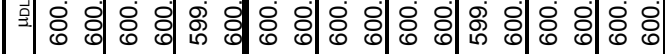

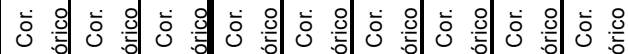

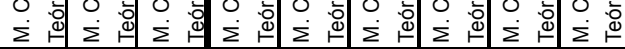

\begin{tabular}{|c|c|c|c|c|c|c|c|c|}
\hline & & & & & & & & \\
\hline$\overline{0}$ & $\begin{array}{l}m \\
0 \\
0 \\
0^{\circ}\end{array}$ & $\begin{array}{l}00 \\
0 \\
0 \\
0 \\
0\end{array}$ & $\begin{array}{l}\overline{0} \\
\overline{11} \\
0^{0}\end{array}$ & $\dddot{0}_{0}^{m}$ & $\begin{array}{l}0 \\
0 \\
11 \\
0 \\
0\end{array}$ & $\bar{i}$ & $\begin{array}{l}0 \\
0 \\
11 \\
0 \\
0\end{array}$ & $\begin{array}{l}0 \\
0 \\
11 \\
0 \\
0 \\
0\end{array}$ \\
\hline \multicolumn{3}{|c|}{$\begin{array}{l}\text { әреp!!euozes } \\
\text { uəs oeș!^əגd }\end{array}$} & \multicolumn{3}{|c|}{$\begin{array}{l}\text { әрер!|euozes } \\
\text { ex!eq } \\
\text { moо оеsı^әд }\end{array}$} & \multicolumn{3}{|c|}{ 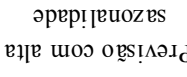 } \\
\hline
\end{tabular}

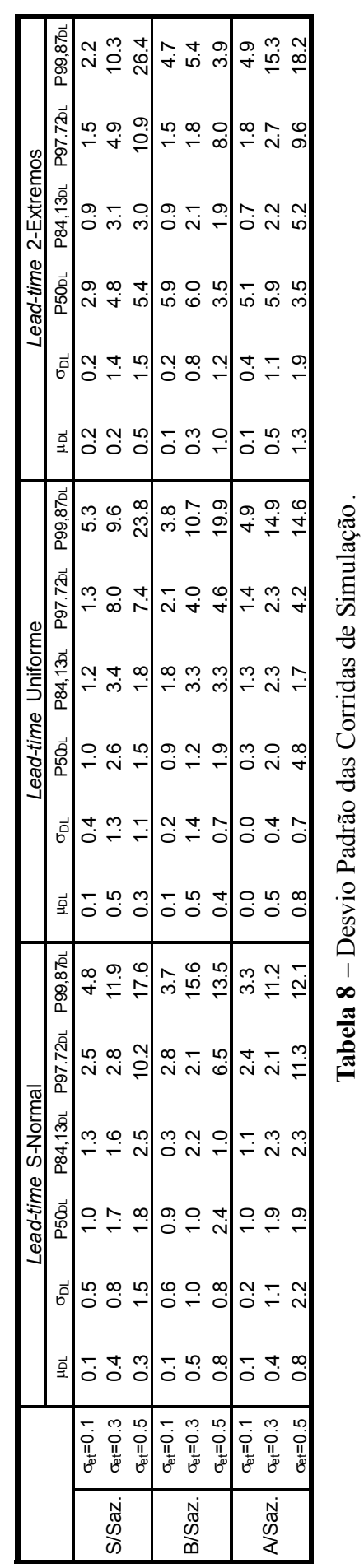



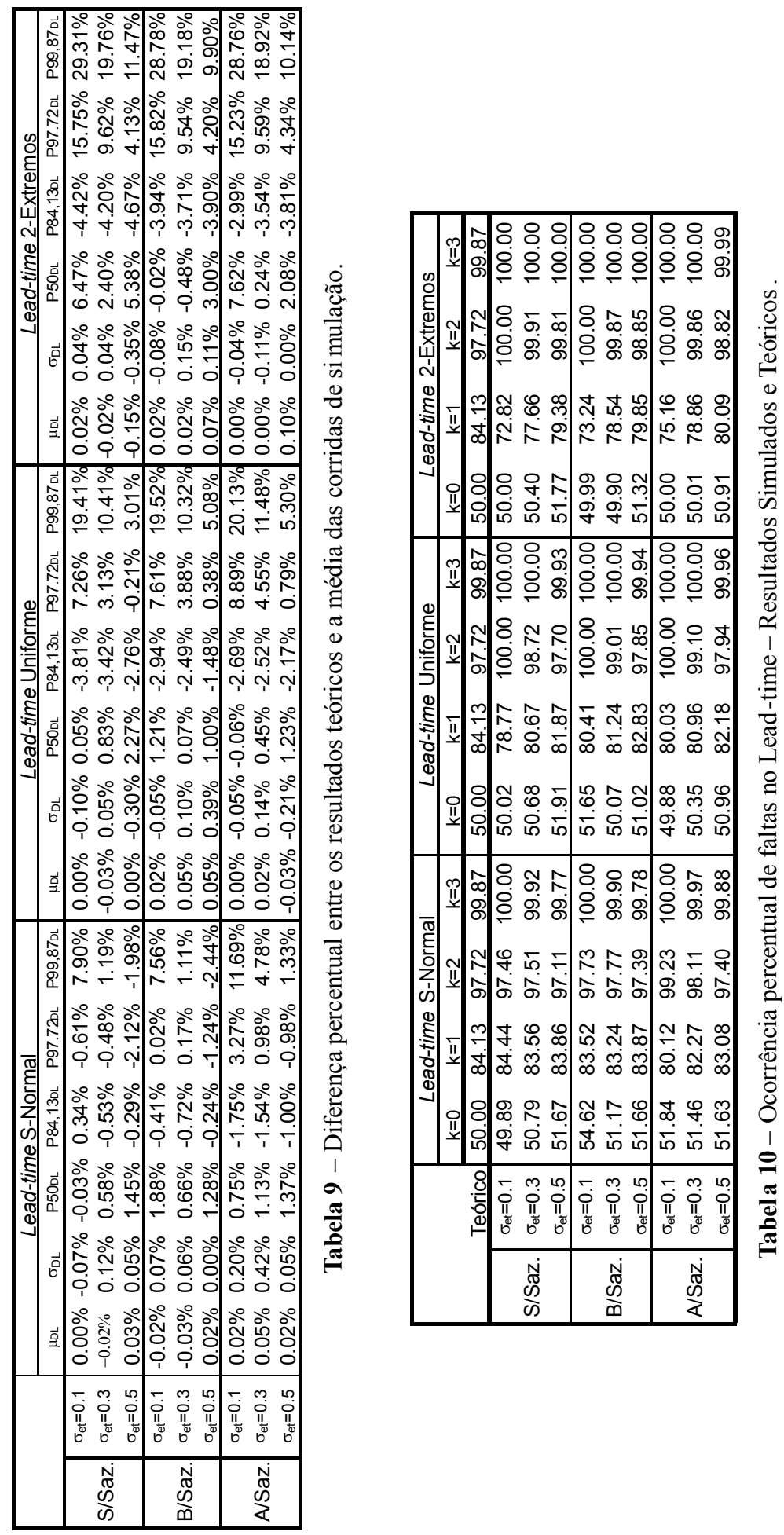

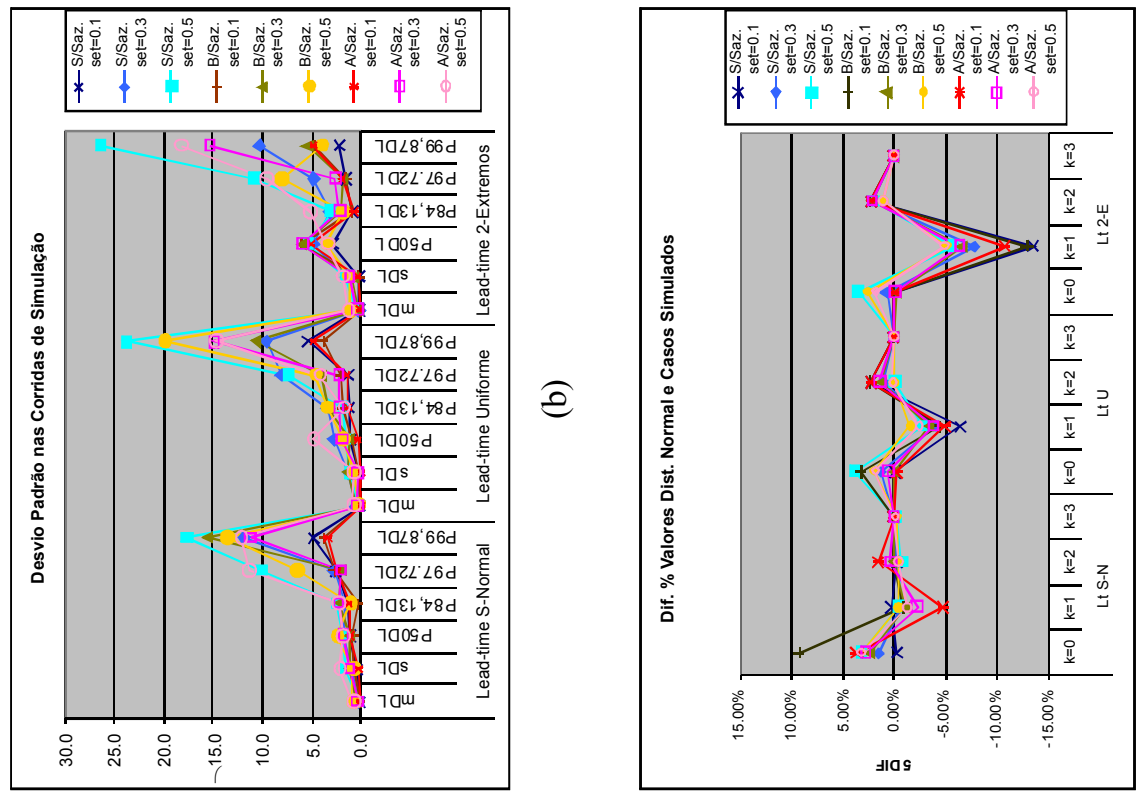

?
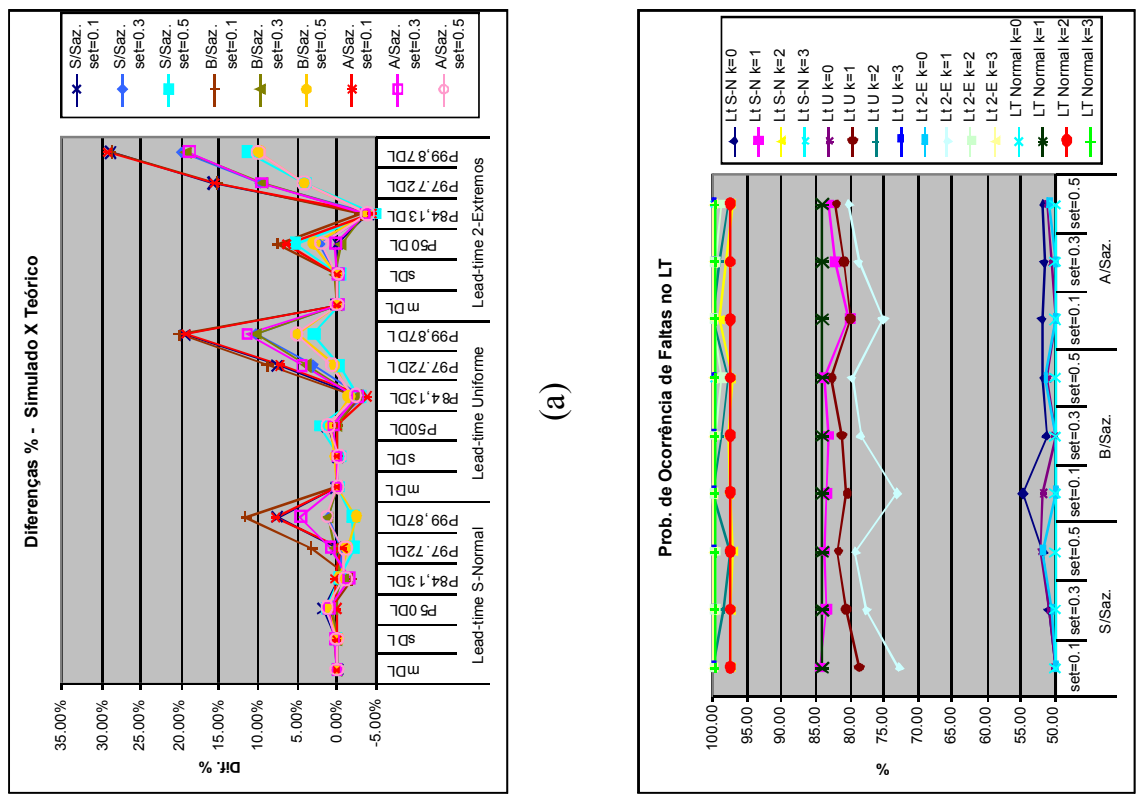

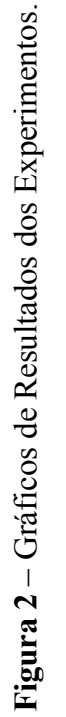




\section{Conclusões}

O ponto de pedido baseado em previsões desenvolvido é uma nova maneira de se gerenciar estoques em ambientes nos quais previsões agregam informações valiosas ao processo de planejamento. Manufaturas usando MRP (Material Requirements Planning) são exemplos de ambientes onde o modelo apresentado pode ser usado. Segerstedt (1996), Hautaniemi \& Pirttila (1999) e Buzacott \& Shanthikumar (1994) são exemplos de artigos que tratam simultaneamente de incertezas na demanda e no lead-time.

Como mostrado ao longo do artigo, o modelo proposto tem muitas vantagens quando comparado a seu análogo clássico. Pode inclusive ser concluído que o novo modelo amplia o escopo e a aplicabilidade do modelo clássico. Se as previsões $f_{i}$ e os parâmetros das variáveis aleatórias $e_{i}$ são iguais em todos os períodos no horizonte de planejamento, $r_{f}$ and $r$ geram os mesmos resultados, ou seja, $r$ pode ser considerado como um caso particular de $r_{f}$ como mostrado no primeiro experimento da simulação.

Outra importante característica de $r_{f}$ é que seu valor pode variar ao longo do tempo, se ajustando a padrões previsíveis da demanda. Tal atributo agrega flexibilidade ao ambiente operacional de uma empresa e pode reduzir custos de carregar estoques e de demandas não atendidas. A figura 3 ilustra a flexibilidade adicionada pelo modelo.

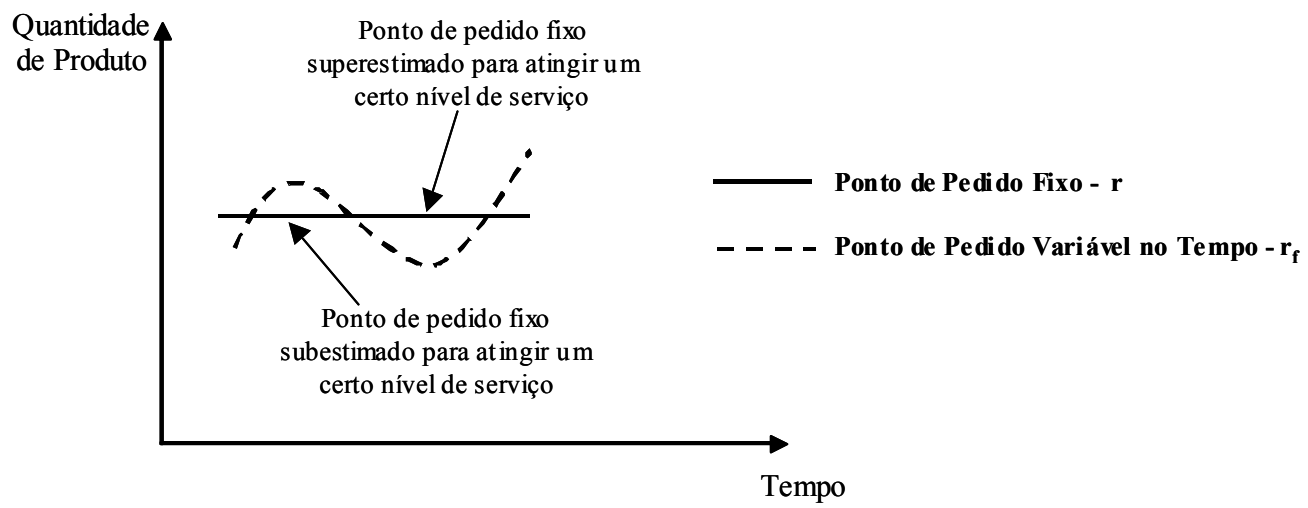

Figura 3 - Flexibilidade trazida pelo modelo proposto.

Como no modelo clássico, uma possível limitação do uso de $r_{f}$ é a premissa de que a demanda no lead-time é normalmente distribuída. Nas simulações realizadas esta premissa não resultou em desvios significativos na maioria dos cenários analisados. Entretanto pode ser interessante utilizar outras distribuições para $D L$, como feito por Namit \& Chen (1999) e Keaton (1995) para o modelo clássico.

Outras possíveis extensões para o modelo proposto são considerar incertezas no processo de produção, como apresentado por Hung \& Chang (1999) e por Gullu, Onol \& Nesim (1999), e ainda ambientes multielos como mostrado em artigos recentes, tais como: Minner (2003), So \& Zheng (2003), Zhang (2004) e Chen et al. (2000). 


\section{Referências Bibliográficas}

(1) Ballou, R.H. (2001). Gerenciamento da Cadeia de Suprimentos. Bookman.

(2) Banks, J. (1999). Discrete-Event System Simulation. Prentice-Hall, Inc., Upper Saddle River, New Jersey.

(3) Bowersox, D.J. \& Closs, D. (1996). Logistical Management. McGraw-Hill, New York.

(4) Brown, R.G. (1967). Decision Rules for Stock Management. Holt, Rinehart and Winston, New York.

(5) Buzacott, J.A. \& Shanthikumar, J.G. (1994). Safety Stocks versus Safety Time in MRP Controlled Production Systems. Management Science, 40(12), 1678-1689.

(6) Chen, F.; Drezner, Z.; Ryan, J.K. \& Simchi-Levi, D. (2000). Quantifying the bullwhip effect in a simple supply chain: The impact of forecasting, lead-times, and information. Management Science, 46, 436-443.

(7) Eppen, G.D. \& Martin, R.K. (1988). Determining Safety Stock in the Presence of Stochastic Lead-time and Demand. Management Science, 34(11), 1380-1390.

(8) Garcia, E.S.; Reis, L.M.T.V.; Machado, L.R. \& Ferreira Filho, V.J.M. (2006). Gestão de Estoques: Otimizando a Logística e a Cadeia de Suprimentos. Rio de Janeiro, e-Papers.

(9) Garcia, E.S.; Silva, C.F. \& Saliby, E. (2002). A Simulation Model to Validate and Evaluate the Adequacy of an Analytical Expression for proper Safety Stock Sizing. Proceedings of the 2002 Winter Simulation Conference.

(10) Gullu, R.; Onol, E. \& Nesim, E. (1999). Analysis of an Inventory System under Supply Uncertainty. International Journal of Production Economics, 59, 377-385.

(11) Hardley, G. \& Within, T.M. (1963). Analysis of Stock Systems. Prentice-Hall Inc., Englewood Cliffs, NJ.

(12) Hautaniemi, P. \& Pirttila, T. (1999). The Choice of Replenishment Policies in an MRP Environment. International Journal of Production Economics, 59, 85-92.

(13) Hung, Y-F \& Chang, C-B. (1999). Determining Safety Stocks for Production Planning in Uncertain Manufacturing. International Journal of Production Economics, 58, 199-208.

(14) Keaton, M. (1995). Using the Gamma Distribution to Model Demand When Lead-time is Random. Journal of Business Logistics, 16(1), 107-131.

(15) Krupp, J.A.G. (1997). Safety Stock Management. Production and Stock Management Journal, third quarter, 11-180.

(16) Lariviere, M. \& Porteus, E. (1999). Stalking information: bayesian inventory management with unobserved lost sales. Management Science, 45(3), 346-363.

(17) Lau, H.-S. (1989). Toward a Stock Control System under Non-Normal Demand and Lead-time Uncertainty. Journal of Business Logistics, 10(1), 88-103.

(18) Mentzer, J.T. \& Krishnan, R. (1988). The effect of the assumption of normality on inventory control/customer Service. Journal of Business Logistics, 6(1), 101-120. 
(19) Meyer, P. (1965). Introductory probability and statistical applications. AddisonWesley Publishing Company, New York.

(20) Minner, S. (2003). Multiple-supplier inventory models in supply chain management: A review. International Journal of Production Economics, 81-82, 265-279.

(21) Namit, K. \& Chen, J. (1999). Solutions to the $<$ Q,r $>$ Inventory Model for Gamma Lead-Time Demand. International Journal of Physical Distribution and Logistics, 29(2), 138-151.

(22) Segerstedt, A. (1996). Formulas of MRP. International Journal of Production Economics, 46-47, 127-136.

(23) Silver, E. (1981). Operations research in inventory management: a review and critique. Operations Research, 29(4), 628-645.

(24) Silver, E.A.; Rycke, D. \& Peterson, R. (1998). Inventory Management and Production Planning and Scheduling. John Wiley \& Sons, New York.

(25) So, K.C. \& Zheng, X. (2003). Impact of supplier's lead-time and forecast demand updating on retailer's order quantity variability in a two-level supply chain. International Journal of Production Economics, 86, 169-179.

(26) Tyworth, J.E. (1992). Modeling Transportation-Stock Trade-offs in a Stochastic Setting. Journal of Business Logistics, 13(2), 97-124.

(27) Wanke, P. \& Saliby, E. (2005). Proposta para a Gestão de Estoques de Novos Produtos: Solução do Modelo (Q,r) para a Distribuição Uniforme da Demanda e do Lead-time de Suprimento. Gestão \& Produção, 12(1), 1-20.

(28) Zhang, X. (2004). The impact of forecasting methods on the bullwhip effect. International Journal of Production Economics, 88, 15-27.

(29) Zinn, W. \& Marmorstein, H. (1990). Comparing Two Alternative Methods of Determining Safety Stock Levels: The Demand and the Forecast Systems. Journal of Business Logistics, 11(1), 95-110.

(30) Zinn, W.; Marmorstein, H. \& Charnes, J. (1992). The Effect of Autocorrelated Demand on Customer Service. Journal of Business Logistics, 13(1), 173-192.

(31) Zipkin, P. (2001). The limits of mass customization. MIT Sloan Management Review, 42(3), 81-87. 